\title{
Hands-Free Manipulation Using Simple Bio-Potential Interface System*
}

\author{
Kazuhiko TAKAHASHI** ${ }^{* *}$, Takashi NAKAUKE*** and Masafumi HASHIMOTO** \\ ** Department of Information Systems Design, Doshisha University \\ 1-3 Miyakodani Tatara, Kyotanabe, Kyoto 610-0321, Japan \\ E-mail: \{katakaha, mhashimo\} @ mail.doshisha.ac.jp \\ *** Nomura Research Institute, Ltd. (ex-affiliation Graduate school of Doshisha University) \\ Marunouchi Kitaguchi Building, 1-6-5 Marunouchi, Chiyoda-ku, Tokyo 100-0005, Japan \\ E-mail: nakauke@gmail.com
}

\begin{abstract}
This paper proposes a nonverbal interface system using bio-potential signals, such as EOG and EMG, measured by a brain-computer interface and investigates its possibility of application to control of a hands-free manipulation system. A simple gesture recognition algorithm is presented to estimate the user's thinking from the EOG and EMG signals. To evaluate the feasibility and the characteristics of the interface system for hands-free manipulation, moving control experiments in 3D virtual space are carried out and the effectiveness of the proposed interface system is confirmed.
\end{abstract}

Key words : BCI, EOG, EMG, EEG, Gesture Recognition, Control

\section{Introduction}

Recently, utilizing computers in our daily life is increasing with progress of information technology and thus communication between humans and machines are becoming more and more common. Nonverbal information, such as facial expression, gaze and gesture, plays an important role in human communication and using it makes human communication smooth. It is clear that the exchange of nonverbal information is important in all forms of communication and is sometimes more important than verbal information. To achieve more intimate and human-like interactions between humans and machines, the use of nonverbal information for human-machine interface will be effective and essential. Furthermore, such an interface will be expected to aid communication for not only ordinary people but also disabled people. Many studies have been undertaken in order to develop communication system between human and machines by utilizing nonverbal information. For example, computer vision technologies especially have widely been applied to recognize nonverbal information ${ }^{(1)(2)}$, such as gestures and sign language, several practical applications are presented in human-machine interface systems, virtual reality applications, and video game systems. Additionally, a multimodal interface, such as combining with audio signals and video images, is effective to recognize nonverbal information ${ }^{(3)}$.

Another possible communication system of utilizing nonverbal information is use of biopotential signals, such as electrooculargraphic (EOG), electromyographic (EMG), and electroencephalographic (EEG). The EOG and EMG signals are caused from the human's physiological changes, however, the human body motions have a relationship with the human's psychological states, such as emotions, intention and thinking, consciously and/or unconsciously. Therefore, it may be possible to estimate the human's thinking if machines could utilize and recognize these signals effectively. From its potentials, many studies of applying the EMG signal to the human-machine interface have been carried out ${ }^{(4)(5)(6)(7)(8)(9)}$. The EOG signal would be also expected as a mode of the interface ${ }^{(10)(11)(12)(14)(13)(15)(16)}$. It would be ideal if such bio-potential signal could be used in the interface system, however there 
have been certain problems when the signal has been used unimodally ${ }^{(17)}$ (e.g., there have been difficulties in finding and selecting objects or commands when the eye movement has been utilized unimodally as the only method for computer input, since degree of freedom for controlling is not enough). On the other hand, estimating emotions or thinking from the EEG signal, which is an index of the central nervous system, seems to be general and effective since emotions and thinking are excited in the limbic system and are deeply related to cognition process. Thus the communication method that links the computer to the human nervous and muscular systems, brain-computer interface (BCI), is expected to have a potential of achieving universal and seamless human-machine interface ${ }^{(18)}$ and many studies have been undertaken $^{(19)(20)(21)(22)(23)(24)(25)(26)}$. Moreover, the communication system using the BCI serves various purposes of communication aid for not only us but also disabled or aged person.

In order to achieve an intelligent human-machine interface system that recognizes nonverbal information of user, this paper proposes a nonverbal interface system using bio-potential signals measured by a simple BCI. A simple gesture recognition algorithm is presented to estimate the user's thinking from the EOG and EMG signals, and a control method that combined EOG gesture recognition result and EMG gesture recognition result multimodally is considered to achieve a hands-free manipulation system. Pilot study of estimating user's thinking from the EEG signal is also investigated. To investigate the effectiveness of utilizing the proposed interface system, it is applied to controlling moving applications in the hands-free manipulation system. In section 2, the hands-free manipulation system using the nonverbal interface system is proposed and the gesture recognition algorithm of the bio-potential signals is presented. The controlling application in a virtual space is described and the results of experiment using proposed interface system are presented in section 3. In section 4, controlling a mobile robot with the hands-free manipulation system is demonstrated as an example of practical application.

\section{Hands-Free Manipulation System}

\subsection{System configuration}

Figure 1 shows the configuration of a hands-free manipulation system. In the system, one personal computer is for an operator to estimate her or his thinking using the BCI (hereafter called operator-PC), and another personal computer is for a controlled object to send control commands or receive sensor information (hereafter called object-PC). All computers are connected via an Ethernetwork. An operator wears the BCI on her or his forehead to collect bio-potential signals, such as EEG signal, EMG signal, and EOG signal. These signals are sent to the operator-PC to generate control commands by recognizing operator's bio-potential signals. In the system, many kinds of hardware, such as a robot manipulator, a mobile robot, an auto-driven wheel chair, and a network camera, can be connected as the controlled objects.

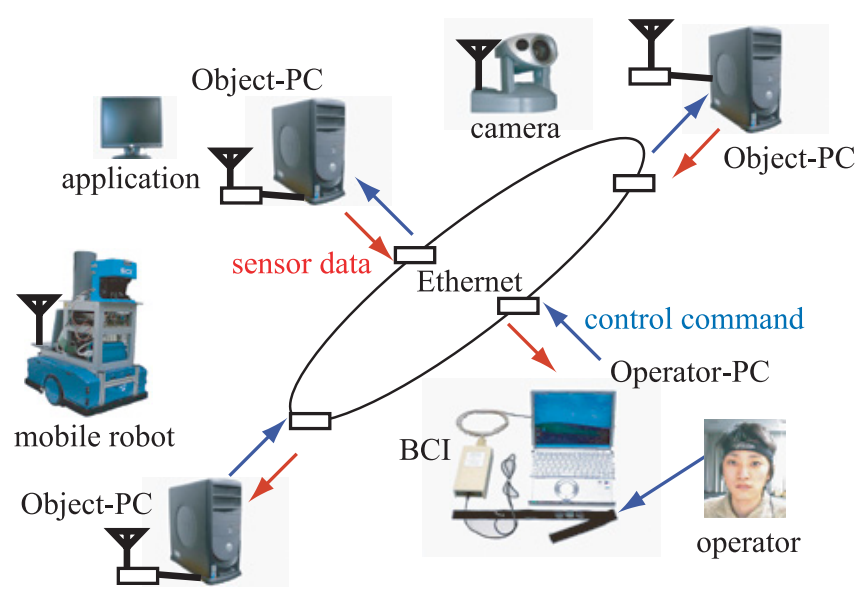

Fig. 1 Overview of hands-free manipulation system. 

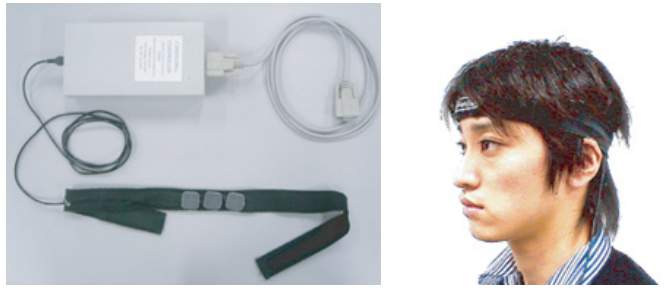

Fig. 2 Simple brain-computer interface.

The control command transmitted from the operator-PC is received by the object-PC and then is sent to the control object via wired and/or wireless communication. The environmental information observed by sensors, such as a CCD camera and a laser range sensor, mounted on the object is also transmitted to the operator-PC from the object-PC via wired and/or wireless communication in real time. For example, in the system, the operator knows the environmental condition around the controlled object by watching the video images and then makes commands by using the BCI how to control the object.

\subsection{BCI}

Figure 2 shows the BCI (The Cyberlink ${ }^{T M}$ Interface, Brain Actuated Technologies, Inc. (27)) used in our proposed hands-free manipulation system. It is optimized to pick up the subject's bio-potential signals from her or his forehead $\left(F_{p 1}\right.$ and $F_{p 2}$ in the augmented 10-20 system) in real time with three dry electrodes that are embedded in the headband to of reduce the cost and protect the human body off from the risks of inserting electrodes. The interface unit has the amplifier that has a gain of 50000 and the filter system that provides a bandpass filter with a bandwidth of $0.5 \mathrm{~Hz}$ to $45 \mathrm{~Hz}$. The gathered signals are separated using frequency domain analysis algorithm ${ }^{(28)}$ into the EEG, EOG, and EMG signals in the interface unit and are then transferred into a personal computer via RS-232C.

\subsection{Gesture recognition from EEG}

Brain activities lead to EEG signals. The final goal of the hands-free manipulation system is to achieve controlling objects by EEG information. To investigate whether it is possible to extract operator's thinking from features of EEG obtained by the simple BCI or not, we carried out experiments of recognizing simple images: e.g., the subject wears the BCI and images her or his body motions, such as lift her or his left or right hand.

The left figure of fig. 6 shows an example of the EEG signal transmitted into the personal computer from the interface unit of the BCI. The sampling time is $1 \mathrm{~ms}$. In this study, the mean of the power ${ }^{(29)}$ for each brain wave component, such as $\theta(4-8 \mathrm{~Hz}), \alpha(8-13 \mathrm{~Hz})$, and $\beta$ (13 - $30 \mathrm{~Hz}$ ) wave bands, is considered as a feature component. After processing by a digital low pass filter (Butterworth filter, cutoff frequency of $30 \mathrm{~Hz}$ ) to the EEG signal, FFT analysis is applied to the EEG signal and then the mean of the power is defined as follows.

$$
P_{i}=\frac{1}{M_{\omega_{i}}} \sum_{\omega_{i}}\left|\sum_{k=0}^{N-1} \frac{x_{e e g}(k)-\mu}{\sqrt{\sigma}} e^{-j \frac{2 \pi \omega k}{N}}\right|
$$

where $i$ indicates $\theta, \alpha$, and $\beta$, respectively, $x_{e e g}(k)$ is the EEG signal, $k$ is the sampling number, $\mu$ and $\sigma$ are the mean and deviation of EEG signal measured under relaxed condition (baseline), respectively, and $M$ is the number of frequency component. The right figure of fig. 6 is the power of the EEG signal shown in the left figure of fig. 3 where $N=256$.

The experiments of imaging were carried out five times by three subjects (Japanese, male). The subject wearing the BCI sat down on a chair and imaged body motions with closing his eyes. The task of imaging body motion was the lifting either left or right hand. A set of measurement is: the EEG signal is collected during first 30 seconds under relaxed condition, and is gathered during next 10 seconds by imaging body motion. Table 1 shows the 

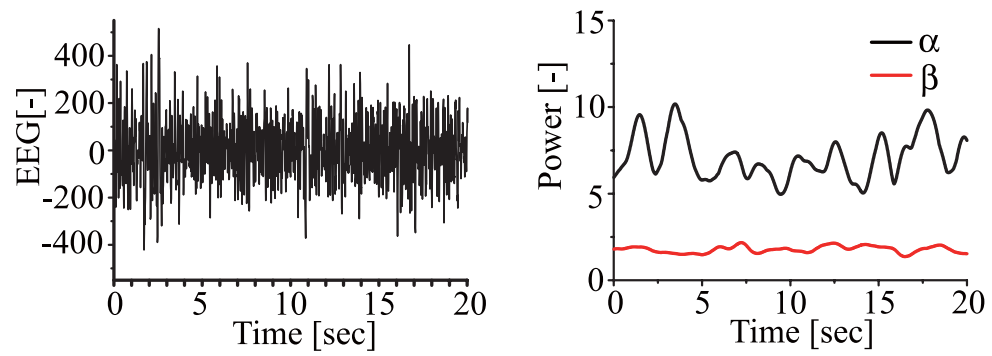

Fig. 3 Example of EEG signal.

Table 1 Power of EEG for imaging body motions.

\begin{tabular}{|c|c|c|c|c|c|c|}
\hline & $P_{\alpha}^{\text {left }}$ & $P_{\beta}^{\text {left }}$ & $P_{\alpha}^{\text {right }}$ & $P_{\beta}^{\text {right }}$ & $P_{\alpha}^{\text {relax }}$ & $P_{\beta}^{\text {relax }}$ \\
\hline Mean & 8.13 & 2.14 & 7.39 & 1.89 & 7.49 & 1.86 \\
\hline Deviation & 0.42 & 0.03 & 0.18 & 0.01 & 0.14 & 0.01 \\
\hline
\end{tabular}

experimental result, however, remarkable differences between two images are not obtained by t-test with $5 \%$ level of statistical significance. Since it is hard to recognize operator's thinking from the EEG signal collected with our simple BCI, the EEG signal is used to observe operator's states ${ }^{(30)}$ : relax or arousal.

\subsection{Gesture recognition from EOG}

Eye motions lead to the EOG signal. Figure 3 shows examples of the EOG signal transmitted into the personal computer from the interface unit. The signal illustrated in the left figure of fig. 4 is obtained when she or he looks to left or right. By using the EOG signal, it is possible to estimate her or his eyes' movement to left or right direction according to the following procedure.

( 1 ) To eliminate noise caused by small eye motions such as blinking and saccade, a dead zone of width $T_{d}$ is defined.

(2) The EOG signal $x_{\text {eog }}(k)$ is transformed into a rectangular signal of amplitude $A_{r}$.

$$
\begin{aligned}
& \text { if } x_{\text {eog }}(k)>T_{d} \\
& \text { then } x_{\text {eog }}(k)=A_{r} \\
& \text { else } x_{\text {eog }}(k)=0
\end{aligned}
$$

( 3 ) By finding falling edges of the rectangular signal, it is transformed into impulse signal $u(k)$ of amplitude $A_{i}$.

(4) By applying a digital integral filter to the impulse signal $u(k)$ twice, a trapezoidal signal $y(k)$ is obtained.

( 5 ) The eye direction can be recognized with thresholds $T_{L}$ and $T_{R}$ by the following algorithm.
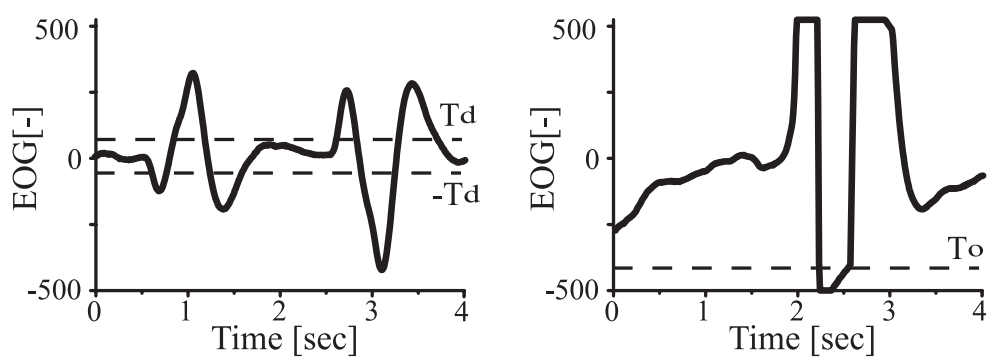

Fig. 4 Examples of EOG signal. 

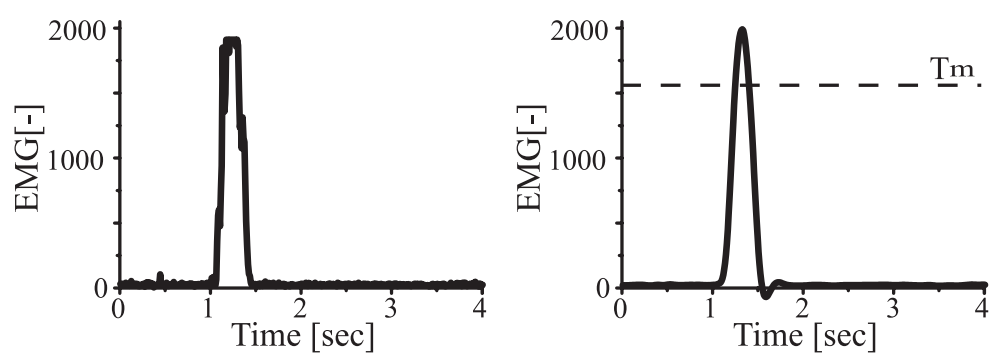

Fig. 5 Example of EMG signal.

if $y(k)>T_{L}$ and $y(k+1) \leq T_{L}$ and $y\left(k+\tau_{o_{1}}\right) \leq T_{L}$ and $y\left(k+\tau_{o_{1}}+1\right)>T_{L}$ then look left

if $y(k)<T_{R}$ and $y(k+1) \geq T_{R}$ and $y\left(k+\tau_{o_{1}}\right) \geq T_{R}$ and $y\left(k+\tau_{o_{1}}+1\right)<T_{R}$ then look right

Here $\tau_{o_{1}}$ is the interval time.

To prevent misrecognition, the integral filter is reset when an constant interval passes after the signal $y(k)$ is obtained or an abnormal value is observed in the signal $y(k)$.

On the other hand, the EOG signal shown in the right figure of fig. 4 is obtained when she or he stares at a certain point and her or his middle of the forehead is wrinkled. This gesture can be easily recognized with a simple threshold $T_{o}$ by the following algorithm.

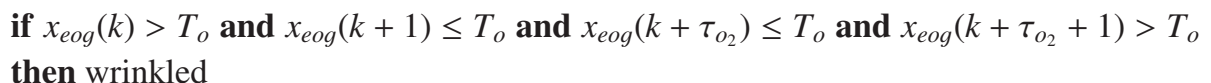

Here $\tau_{o_{2}}$ is the interval time. This gesture recognition has a person independent characteristic.

\subsection{Gesture recognition from EMG}

Facial muscle motions lead to the EMG signal. The left figure of fig. 5 shows an example of the EMG signal obtained when she or he closes her or his jaw. Since a noise is put on the EMG signal, the digital low pass filter (Butterworth filter, cutoff frequency of $4 \mathrm{~Hz}$ ) is applied to the EMG signal as shown in the right figure of fig. 5. By using simple threshold algorithm to the EMG signal $x_{e m g}(k)$, the jaw gesture can be recognized by the following algorithm.

$$
\begin{aligned}
& \text { if } x_{e m g}(k) \leq T_{m} \text { and } x_{e m g}(k+1)>T_{m} \text { and } x_{e m g}\left(k+\tau_{m}\right)>T_{m} \text { and } x_{e m g}\left(k+\tau_{m}+1\right) \leq T_{m} \\
& \text { then jaw closed }
\end{aligned}
$$

Here $T_{m}$ is the threshold and $\tau_{m}$ is the interval time. This gesture recognition has also a person independent characteristic.

\subsection{Control algorithm application}

In order to achieve the hands-free manipulation using the gesture recognition results of the bio-potential signals obtained by the BCI, it is important to consider the combination of the EOG and EMG signals. In manipulation systems, the number of states which should be controlled depends on the control object generally. By using the gesture recognition result of EOG signal proposed in the section 2.4, four states (left/right, on/off) can be controlled. Although two states (on/off) can be controller by using the gesture recognition result of EMG signal proposed in the section 2.5, a gesture of twice jaw closing as a double click motion is 
Table 2 Relationship between gesture and control command.

\begin{tabular}{|c|c|c|}
\hline Control command & EOG & EMG \\
\hline Go forward & - & single click \\
\hline Go backward & - & double click \\
\hline Turn left & look left & - \\
\hline Turn right & look right & - \\
\hline Stop & single click & - \\
\hline
\end{tabular}

introduced to increase the degree of freedom in control. Assuming of controlling a mobile robot in a physical space or controlling a walk-through in a virtual space, a state control algorithm by using the EOG and EMG signals is proposed as shown in table 2.

\section{Hands-Free Manipulation Experiment}

To investigate the feasibility of the gesture recognition algorithm and evaluate the usefulness of applying it in practical control applications of hands-free manipulation, we carried out control experiments using a walk through application in the virtual space. The walk through application is that she or he tries to move toward a destination point while avoiding obstacles arranged at random in the 3D virtual space. The moving speed in the virtual space is a constant value to simplify the control process.

Figure 6 shows the experimental setup that is composed with the BCI, two personal computers (DELL DIMENSION 8400, Pentium 4, 3.2GHz, Windows XP SP2), and a rear projection monitor (EPSON ELS-65GL1H). The recognition and control algorithms are implemented with C language (Visual Studio.NET) into the operator-PC. The application of walk through in the 3D virtual space is programmed by using Open GL graphic system and is implemented into the object-PC. These computers are connected via 100 base-T EthernetWork.

The control experiment was carried out in the following manner.

( 1 ) Calibration: Some threshold parameters are tuned depending on subject.

(2) Training: The subject tries a training task without considering any limitation. The training is freely walk through in the virtual space in order to know the relationship between the gestures and control commands.

( 3 ) Test: The subject tries the walk through task. The task is finished when the subject can get a score of 150 where each box object placed randomly in the virtual space has a different score. When the subject touches to the object, she or he can get the score. While the subject carries out the task, the number of times that the subject makes control commands is counted, the traveling time that the subject completes the task is measured, and the EEG signal is also recorded.

( 4 ) Evaluation: The operability of the interface system and its comfortableness are evaluated by using questionnaires based on the semantic differential technique.

The experiment was done once per day by 11 subjects of our laboratory (Japanese, 10 males and one female) for five days. The threshold parameters in the recognition algorithm

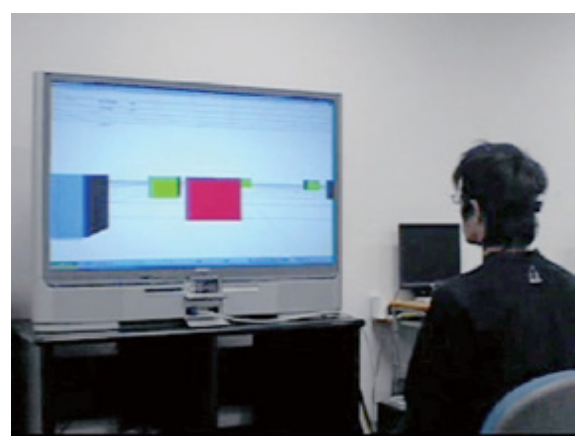

Fig. 6 Experimental setup of walk through in 3D virtual space. 


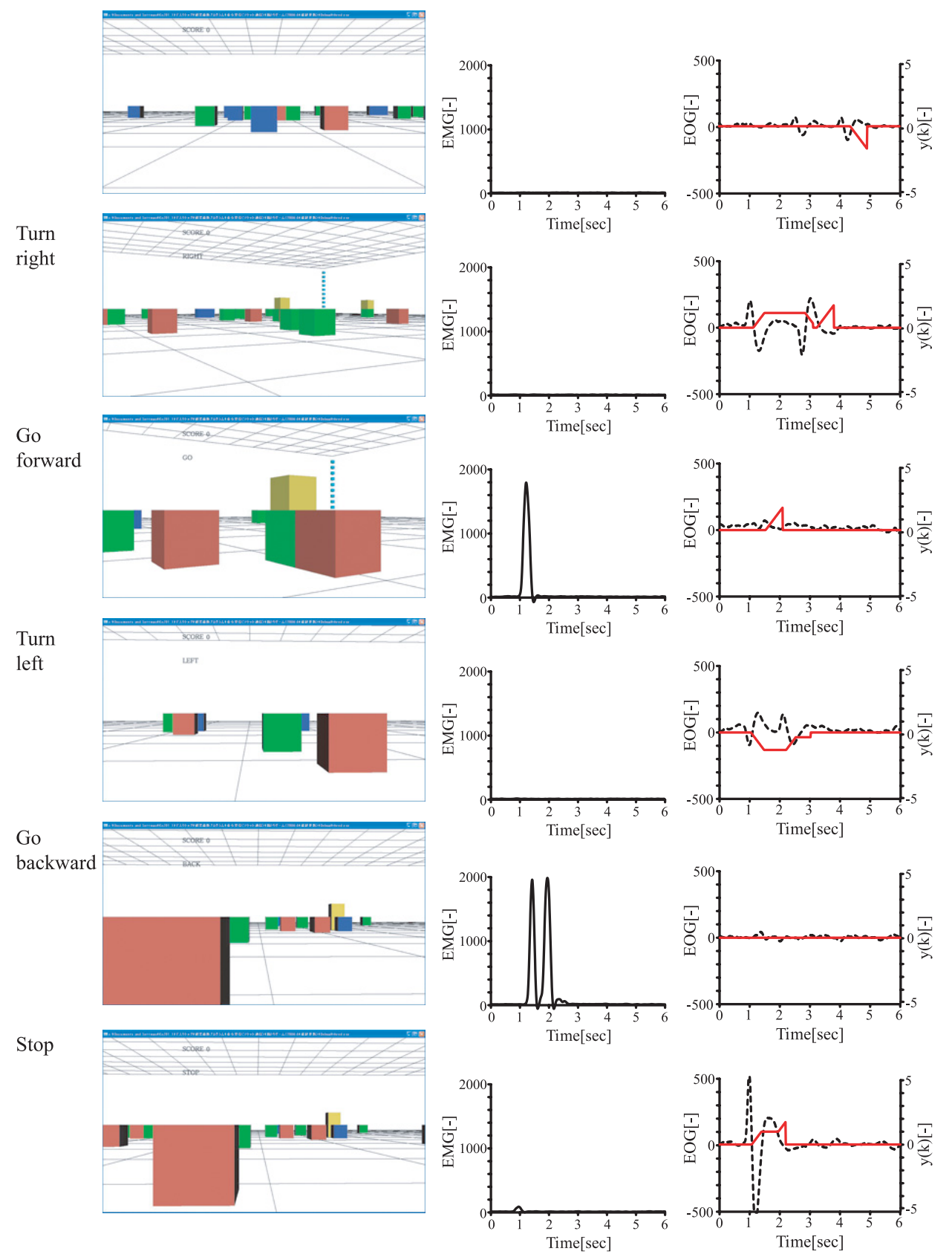

Fig. 7 Example of experimental sequence.

were set to $T_{L}=-0.4, T_{R}=0.4, \tau_{o_{1}}=200, T_{o}=-450, \tau_{o_{2}}=150, T_{m}=900$, and $\tau_{m}=150$. These values were defined by trial and error and were in common for all subjects. The initial value of the dead zone width was defined by the following equation, and the value of $T_{d}$ was then tuned manually according to the subject.

$$
T_{d}=\frac{1}{2} \sum_{i=1}^{3}\left[\frac{1}{4} \sum_{j=L, R}\left\{\left|\max \left\{x_{\text {eog }_{j}}(k)\right\}\right|+\left|\min \left\{x_{\text {eog }}(k)\right\}\right|\right\}\right]
$$

Figure 7 shows an example of experiment's sequence where the top figure shows the initial state and the bottom figure indicates the final state. The left image shows the virtual space and the right figure shows the EMG and EOG signals. As shown in fig. 7, the experimental task is completed successfully by recognizing of the EOG and EMG signals. Figure 8 shows the traveling time and the frequency of command. Here these indexes are the averages of 

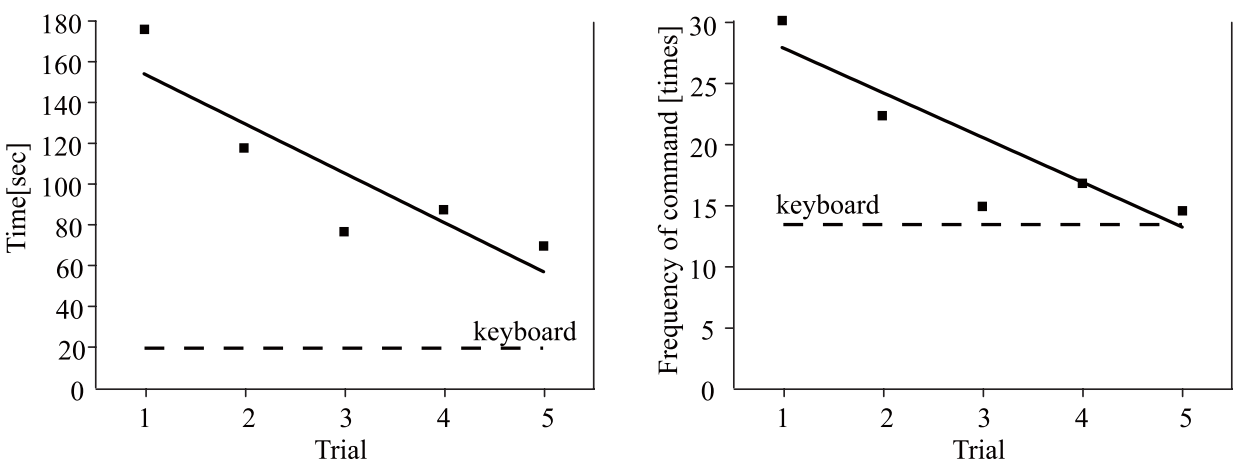

Fig. 8 Experimental results (left: traveling time, right: frequency of command).

Table 3 Recognition rates of control commands.

\begin{tabular}{|c|c|c|c|}
\hline Command & Recognition & Misrecognition & Fail \\
\hline Go forward & 97.3 & 0.9 & 1.8 \\
\hline Go backward & 97.3 & 0.9 & 1.8 \\
\hline Turn left & 83.6 & 0.9 & 15.5 \\
\hline Turn right & 74.6 & 2.7 & 22.7 \\
\hline Stop & 93.6 & 0 & 6.4 \\
\hline
\end{tabular}

Table 4 Result of factor analysis for interface system evaluation.

\begin{tabular}{|c|c|c|c|}
\hline Evaluation scale & Factor 1 & Factor 2 & Factor 3 \\
\hline useful & 0.77 & 0.09 & 0.33 \\
easy & 0.76 & 0.12 & 0.38 \\
comfortable & 0.76 & 0.40 & 0.11 \\
fine & 0.69 & 0.46 & 0.09 \\
interesting & 0.16 & 0.85 & 0.25 \\
enjoyable & 0.41 & 0.71 & 0.39 \\
comprehensible & 0.23 & 0.26 & 0.93 \\
simple & 0.33 & 0.38 & 0.64 \\
\hline Eigenvalue & 4.63 & 0.82 & 0.64 \\
\hline Accumulative contribution rates & 0.32 & 0.55 & 0.76 \\
\hline
\end{tabular}

all subjects. As shown in fig. 8, both of the traveling time and frequency of command decrease as the trial of control experiment progresses. As a reference for comparing the results of proposed interface system, controlling experiments by using the conventional keyboard interface were carried out. Although both indexes are not better rather than those obtained by using the keyboard interface, this result shows that the subjects became used to operation of the interface system and shows the feasibility of using both gesture recognition and control algorithms. In order to evaluate the recognition algorithm, gesture recognition experiments were carried out after 5th trial. Table 3 shows the recognition rates of each control command where the recognition rates are the averages of all subjects. In the recognition experiments, each subject did the gesture ten times for each of the five commands. The average recognition rate is $89 \%$. This result confirms the effectiveness of the recognition algorithm for the EOG and EMG signals.

In the questionnaire, 8 evaluation scales were evaluated by 7 grades between "very good" (score: 7) and "very bad" (score: 1). By applying factor analysis to the results of the questionnaire, three factors were extracted as shown in Table 4 . The factor 1 is an evaluation factor that describes the usefulness of the interface system, the factor 2 is an activity factor that shows the fun of the interface system, and the factor 3 is a potency factor that indicates the easiness of the interface system. The questionnaire was reevaluated by using the factors. Figure 9 shows the average score of each factor. The factors 1 and 3 increase as the trial of control experiment progresses while the factor 2 is almost constant. Thus the operability increases since the subject becomes good at to use the interface system, and the comfortableness has no change from the impression felt at the first trial of the experiment. This results indicates that 

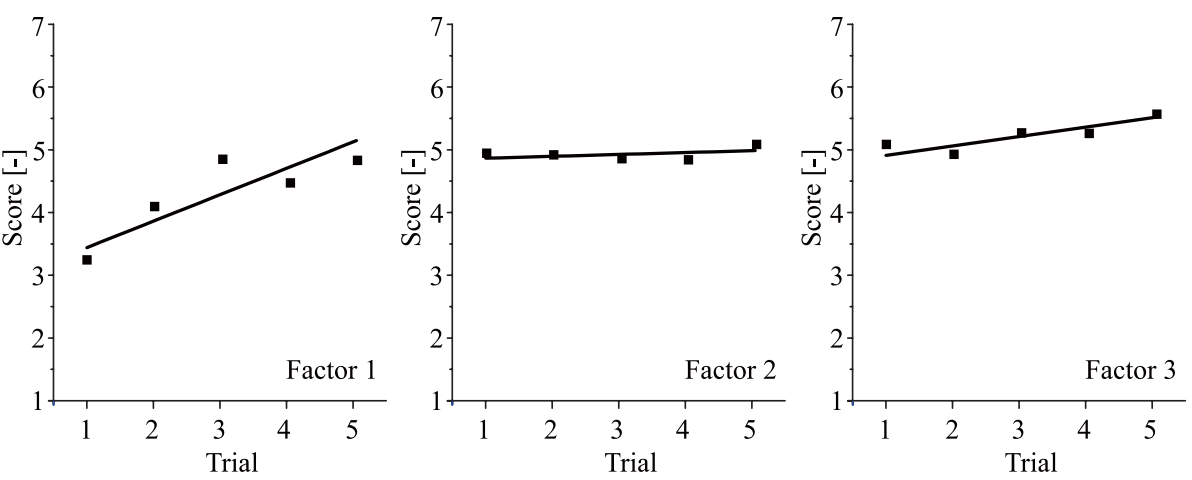

Fig. 9 Evaluation result of questionnaire.

the usefulness of the proposed interface system.

\section{Application to Controlling Mechanical System}

As an example of the hans-free manipulation using the proposed interface system in real world, a mobile robot is applied to the controlled object. The mobile robot composes of a wheeled mobile platform (PATNA Leo-1), a laser range sensor (SICK LMS200), a fiber optical gyro sensor (TAMAGAWA SEIKI, TA7319N3), a CCD camera (SONY EVI-D100), and a personal computer (PANASONIC CF-W4, Pentium M, 1.2GHz, Windows XP SP2) which is used as the object-PC. The operator-PC is the same as the walk through in $3 \mathrm{D}$ virtual space. A network camera (CANON VB-C50i) is utilized to observe motions of the mobile robot. In this application, the operator watches the images sent from the CCD camera mounted
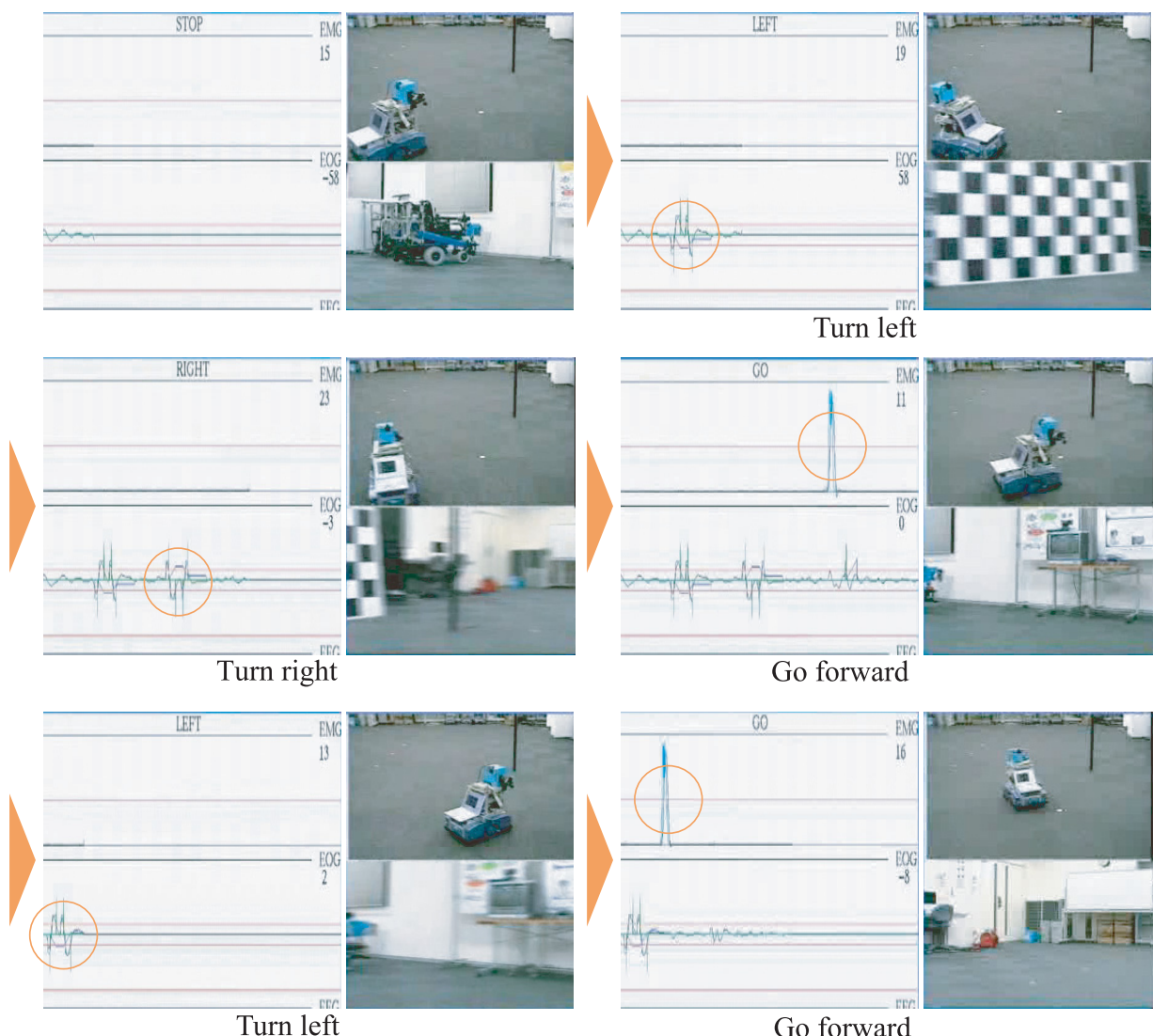

Fig. 10 Example of hands-free manipulation for controlling mobile robot. 
on the mobile robot through the network and makes decisions how to control the mobile robot. The control commands are generated by using our proposed algorithm shown in Table 2 and are sent to the mobile robot via the network.

Figure 10 shows examples of controlling the mobile robot with the hands-free manipulation system. The experiment was carried out in our laboratory. In left figures of each image, the top figure and the bottom figure show the EMG and EOG signals, respectively. The moment of gesture recognition is indicated with the circle in each figure and the generated command is shown under the figure. In right images of each image, the top image shows the mobile robot's motion captured by the network camera while the bottom image is obtained by the onboard CCD camera. In this example, the subject generated the control command in the order of 'turn left', 'turn right', 'do forward', 'turn left', 'go forward'. As shown, the controlling of the mobile robot was successful. These results demonstrate the effectiveness of the interface system for the hands-free manipulation with application to a mechanical system in the real world.

\section{Conclusions}

This paper proposed a nonverbal interface system using bio-potential signals measured with a simple BCI. A simple gesture recognition algorithm is presented to estimate the user's intentions/thougjts from the EOG and EMG signals. To investigate the effectiveness of utilizing the proposed interface system, it is applied to control a moving application in 3D virtual space on the hands-free manipulation system. Experimental results confirmed the feasibility and usefulness of the interface system.

In future works, recognition of user's thinking from the EEG signal should be investigated and utilizing that to control algorithms would be considered.

\section{Acknowledgement}

This study was supported in part by the Academic Frontier Research Project on " New Frontier of Biomedical Engineering Research " of Ministry of Education, Culture, Sports, Science and Technology.

\section{References}

( 1 ) D. M. Gavrila, "The Visual Analysis of Human Movement: A Survey", Computer Vision and Image Understanding, Vol. 73, No. 1 (1999), pp. 82-98.

( 2 ) T. B. Moeslund and E. Granum, "A Survey of Computer Vision-Based Human Motion Capture", Computer Vision and Image Understanding, Vol. 81, No. 3 (2001), pp. 231268.

( 3 ) S. Oviatt, et. al., "Multimodal Interface that Flex, Adapt, and Persist", Communication of the ACM, Vol. 47, No. 1 (2004), pp. 30-75.

( 4 ) A. B. Barreto, S. D. Scargle, and M. Adjouadi, "A Practical EMG-based HumanComputer Interface For Users With Motor Disabilities", Journal of Rehabilitation Research and Development, Vol. 37 No. 1 (2000), pp. 53-64.

( 5 ) K. Coleman, "Electromyography based Human-Computer-Interface to Induce Movement in Elderly Persons with Movement Impairments", Proceedings of the 2001 EC/NSF Workshop on Universal Accessibility of Ubiquitous Computing: Providing for the Elderly, (2001), pp. 75-79.

( 6 ) J. Laakso, M. Juhola, V. Surakka, A. Aula, and T. Partala, "Neural Network and Wavelet Recognition of Facial Electromyographic Signals", Proceedings of the 10th World Congress on Health and Medical Informatics, (2001), pp. 489-492.

( 7 ) O. Fukuda, T.Tsuji, M. Kaneko and A. Otsuka, "A Human-Assisting Manipulator Teleoperated by EMG Signals and Arm Motions", IEEE Transactions on Robotics and Automation, Vol. 19, No. 2 (2003), pp. 210-222.

( 8 ) L. J. Trejo, K. R. Wheeler, C. C. Jorgensen, R. Rosipal, S. T. Clanton, B. Matthews, A. 
D. Hibbs, R. Matthews, and M. Krupka, "Multimodal Neuroelectric Interface Development", IEEE Transactions on Neural Systems and Rehabilitation Engineering, Vol. 11, No. 2 (2003), pp. 199-203.

( 9 ) K. Sagawa and O. Kimura, "Control of Robot Manipulator Using EMG Generated from Face", Proceedings of SPIE, Vol. 6042 (2005), CDROM-604233.

(10) Y. Kuno, T. Yagi, and Y. Uchikawa, "The Development of the Robot Controlling System Using EOG", Proceedings of 7th International Conference on Human-Computer Interaction, (1997), p.44.

(11) H. A. Yanco and J. Gips, "Preliminary Investigation of a Semi-Autonomous Robotic Wheelchair Directed Through Electrodes", Proceedings of the Rehabilitation Engineering and Assistive Technology Society of North America Annual Conference, (1997), pp. 414-416.

(12) J. T. Tecce, J. Gips, C. P. Olivieri, L. J. Pok, and M. R. Consiglio, ’Eye Movement Control of Computer Functions", International Journal of Psychophysiology, Vol. 29, (1998), pp. 319-325.

(13) C. Kyoung and M. Sasaki, "Mobile Robot Control by Neural Network EOG Gesture Recognition”, Proceedings of 8th International Conference on Neural Information Processing, (2001), CDROM-ID225.

(14) R. Barea, L. Boquete, M. Mazo, and E. Lopez, "Wheelchair Guidance Strategies Using EOG”, Journal of Intelligent and Robotic Systems, Vol. 34, (2002), pp.279-299.

(15) M. Williams and R. Kirsch, "Feasibility of Electroculography as a Command Interface for a High Tetraplegia Neural Prosthesis", Proceedings of the 10th Annual Conference of the International Functional Electrical Stimulation Society, (2005).

(16) R. C. Simpson, "Smart Wheelchairs: A Literature Review", Journal of Rehabilitation Research E Development, Vol. 42, No. 4 (2005), pp. 423-438.

(17) V. Surakka, M. Illi, and P. Isokoski, "Gazing and Frowing as a New Human-Computer Interaction Technique", ACM Transactions on Applied Perceptions, Vol. 1, No. 1 (2004), pp. 40-56.

(18) J. Charles, "Neural Interface Link the Mind and the Machine," IEEE Computer Magazine, (1999-1), pp.16-18.

(19) J. R. Walpaw and D. J. McFarland, "Multichannel EEG-Based Brain Computer Communication”, Electrocephalography and Clinical Neurophysiology, Vo. 90, (1994), pp. 444-449.

(20) J. R. LaCourse and E. Wilson, "Brainiac: A Brain-Computer Link", Proceedings of the IEEE Instrumentation and Measurement Technology Conference, (1995), pp. 587-592.

(21) J. R. Millan, J. Mourino, M. G. Marciani, F. Babiloni, F. Topani, I. Canale, J. Heikkonen, and K. Kaski, "Adaptive brain interfaces for physically-disabled people", Proceedings of 20th Annual Internaional Conference of the IEEE Engineering in Medicine and Biology Society, Vol. 4, (1998), pp.2008-2011.

(22) W. D. Penny, S. J. Roberts, E. A. Curran, and M. J. Stokes, ”EEG-based Communication: A Pattern Recognition Approach", IEEE Transactions on Neural Systems and Rehabilitation Engineering, Vol. 8 (2000), pp. 214-215.

(23) E. Curran, P. Sykacek, M. Stokes, S. J. Roberts, W. Penny, I. Johnsrude, and A. M. Owen, "Cognitive Tasks for Driving a Brain-Computer Interfacing System: A Pilot Study", IEEE Transactions on Neural Systems and Rehabilitation Engineering, Vol. 12, No. 1 (2003), pp. 48-54.

(24) K. Tanaka, K. Matsunaga, N. Kanamori, S. Hori, and H. O. Wang, "Electroencephalogram-based Control of a Mobile Robot", Proceedings of 2003 IEEE International Symposium on Computational Intelligence in Robotics and Automation, (2003), pp. 670-675.

(25) G. E. Fabiani, D. J. McFarland, J. R. Wolpaw, and G. Pfurtscheller, "Conversion of EEG Activity Into Cursor Movement by a Brain-Computer Interface (BCI)", IEEE Trans- 
actions on Neural Systems and Rehabilitation Engineering, Vol. 12, No. 3 (2004), pp. 331-338.

(26) G. Pfurtscheller, R. Leeb, C. Keinrath, D. Friedman, C. Neuper, C. Guger, and M. Slater, "Walking from Thought", Brain Research, Vol. 1071, No. 1 (2006), pp. 145-152.

(27) http://www.brainfingers.com/

(28) A. Junker, BRAIN-BODY ACTUATED SYSTEM, United States Patent 5692517 (1997).

(29) T. Toshima and H. Seiwa, Experiment Manual for Psychology, (1993), Kitaohji Shobou (in Japanese).

(30) K. Takahashi, T. Nakauke, and M. Hashimoto, "Remarks on Hands-Free Manipulation System Using Bio-Potential Signals From Simple Brain-Computer Interface", Proceedings of IEEE International Conference on Systems Man and Cybernetics, (2006), pp.1642-1647. 